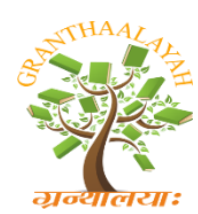
\author{
GRANTHAALAYAH \\ A knowledge Repository
}

INTERNATIONAL JOURNAL OF RESEARCH -

InfoBase Index IBI Factor 3.86

Science

\title{
REDUCTION OF REAL POWER LOSS BY IMPROVED QUANTUM ALGORITHM
}

\author{
Dr.K.Lenin *1 \\ ${ }^{* 1}$ Researcher, Jawaharlal Nehru Technological University Kukatpally, Hyderabad 500 085, India
}

DOI: https://doi.org/10.29121/granthaalayah.v5.i6.2017.2095

\begin{abstract}
In this paper, combination of the Q-bit evolutionary search - quantum particle swarm optimization (QPSO) algorithm and binary bit evolutionary search - genetic particle swarm optimization (GPSO) has been done to solve the reactive power problem \& termed as Improved Quantum Algorithm (IQA). Proposed IQA can be viewed as a kind of hybridization of microspace based search and macro-space based search, which augments the penetrating behavior to augment and balance the exploration and exploitation aptitudes in the whole searching space. In order to evaluate the performance of the proposed IQA algorithm, it has been tested on IEEE 57,118 bus systems and compared to other standard algorithms.
\end{abstract}

Keywords: Quantum Particle Swarm Optimization; Genetic Particle Swarm Optimization; Reactive Power; Transmission Loss.

Cite This Article: Dr.K.Lenin. (2017). "REDUCTION OF REAL POWER LOSS BY IMPROVED QUANTUM ALGORITHM.” International Journal of Research - Granthaalayah, 5(6), 627-636. https://doi.org/10.29121/granthaalayah.v5.i6.2017.2095.

\section{Introduction}

Various mathematical techniques have been adopted to solve this optimal reactive power dispatch problem. These include the gradient method [1, 2], Newton method [3] and linear programming [4-7].The gradient and Newton methods suffer from the difficulty in handling inequality constraints. To apply linear programming, the input- output function is to be expressed as a set of linear functions which may lead to loss of accuracy. Recently Global Optimization techniques such as genetic algorithms have been proposed to solve the reactive power flow problem [8-14]. PSO is inspired by observing the bird flocking or fish school [15]. A large number of birds/fishes flock synchronously, change direction suddenly, and scatter and regroup together. Each individual, called a particle, benefits from the experience of its own and that of the other members of the swarm during the search for food. Comparing with genetic algorithm, the advantages of PSO lie on its simple concept, easy implementation and quick convergence. The PSO has been applied successfully to continuous nonlinear function [15], neural network 
[16], nonlinear constrained optimization problems [17], etc. Most of the applications have been concentrated on solving continuous optimization problems [18]. To solve discrete (combinatorial) optimization problems, Kennedy and Eberhart [19] also developed a discrete version of PSO (DPSO), which however has seldom been utilized. DPSO essentially differs from the original (or continuous) PSO in two characteristics. First, the particle is composed of the binary variable. Second, the velocity must be transformed into the change of probability, which is the chance of the binary variable taking the value one. Furthermore, the relationships between the DPSO parameters differ from normal continuous PSO algorithms [20, 21]. Though it has been proved the DPSO can also be used in discrete optimization as a common optimization method, it is not as effective as in continuous optimization. When dealing with integer variables, DPSO sometimes are easily trapped into local minima [19]. Therefore, Yang et al. [22] proposed a quantum particle swarm optimization (QPSO) for discrete optimization in 2004. Their simulation results showed that the performance of the QPSO was better than DPSO and genetic algorithm. Recently, Yin [23] proposed a genetic particle swarm optimization (GPSO) with genetic reproduction mechanisms, namely crossover and mutation to facilitate the applicability of PSO to combinatorial optimization problem, and the results showed that the GPSO outperformed the DPSO for combinatorial optimization problems. QPSO uses a Q-bit, defined as the smallest unit of information, for the probabilistic representation and a Q-bit individual as a string of Q-bits. The Q-bit individual has the advantage that it can represent a linear superposition of states (binary solutions) in search space probabilistically [22, 24]. Thus the Qbit representation has a better characteristic of population diversity than other representations. However, the performance of simple quantum-inspired PSO is often not satisfactory and is easy to be trapped in local optima so as to be premature convergence. In the binary genetic particle swarm optimization, genetic reproduction, in particular, crossover and mutation, have been combined to form a discrete version particle swarm optimization, is suitable for solving combinatorial optimization problems. In QPSO, the representation of population is Q-bit and evolutionary search is in micro-space (Q-bit based representation space). Differently, in GPSO the representation is binary number and evolutionary search is in macro-space (binary space). It is quite different between QPSO and GPSO in terms of representation and evolution operators. However, as QPSO, the performance of GPSO is also often not satisfactory and is easy to be trapped in local optima so as to be premature convergence. In contrast to the continuous PSO algorithm that has been widely studied and improved by a large body of researchers, the discrete PSO and its application to combinatorial optimization problems has not been as popular or widely studied. Therefore, it is an important topic to develop a new or improved discrete particle swarm optimization algorithm with applications to combinatorial optimization problems. In this paper, combination of the Q-bit evolutionary search - quantum particle swarm optimization (QPSO) algorithm and binary bit evolutionary search - genetic particle swarm optimization (GPSO) has been done to solve the reactive power problem \& termed as Improved Quantum Algorithm (IQA). The performance of (IQA) has been evaluated in standard IEEE 57,118 bus test systems and the results analysis shows that our proposed approach outperforms all approaches investigated in this paper. 


\section{Objective Function}

Active power loss

Main aim of the reactive power dispatch problem is to reduce the active power loss in the transmission network, which can be described as:

$F=P L=\sum_{k \in N b r} g_{k}\left(V_{i}^{2}+V_{j}^{2}-2 V_{i} V_{j} \cos \theta_{i j}\right)$

Where $g_{k}$ : is the conductance of branch between nodes $i$ and $j$, Nbr: is the total number of transmission lines in power systems.

\section{Voltage profile improvement}

For minimization of the voltage deviation in PQ buses, the objective function turns into:

$F=P L+\omega_{v} \times V D$

Where $\omega_{\mathrm{v}}$ : is a weighting factor of voltage deviation.

VD is the voltage deviation given by:

$V D=\sum_{i=1}^{N p q}\left|V_{i}-1\right|$

\section{Equality Constraint}

The equality constraint of the Reactive power problem is represented by the power balance equation, and can be written as, where the total power generation must cover the total power demand and total power loss:

$P_{G}=P_{D}+P_{L}$

Where, $P_{G}$ - Total Power Generation, $P_{D}$-Total Power Demand, $P_{L}-$ Total Power Loss.

\section{Inequality Constraints}

Inequality constraints define the limitations in power system components and power system security. Upper and lower bounds on the active power of slack bus, and reactive power of generators are written as follows:

$P_{\text {gslack }}^{\min } \leq P_{\text {gslack }} \leq P_{\text {gslack }}^{\max }$

$Q_{g i}^{\min } \leq Q_{g i} \leq Q_{g i}^{\max }, i \in N_{g}$

Upper and lower bounds on the bus voltage magnitudes are described as follows:

$V_{i}^{\min } \leq V_{i} \leq V_{i}^{\max }, i \in N$

Upper and lower bounds on the transformers tap ratios are given as follows:

$T_{i}^{\text {min }} \leq T_{i} \leq T_{i}^{\max }, i \in N_{T}$

Upper and lower bounds on the compensators reactive powers are written as follows:

$Q_{c}^{\min } \leq Q_{c} \leq Q_{C}^{\max }, i \in N_{C}$ 
Where $\mathrm{N}$ is the total number of buses, $\mathrm{N}_{\mathrm{T}}$ is the total number of Transformers; $\mathrm{N}_{\mathrm{c}}$ is the total number of shunt reactive compensators.

\section{Quantum Particle Swarm Optimization (QPSO)}

In the quantum theory, the minimum unit that carries information is a Q-bit, which can be in any superposition of state 0 and 1. Let $Q i(t)=\left(q_{i 1}(t), q_{i 2}(t), . ., q_{i D}(t)\right), q_{i d}(t) \in[0,1]$, be quantum particle $I$ with $D$ bits at iteration $t$, where $q_{i d}(t)$ represents the probability of $d$-th bit of $i$-th particle being 0 at iteration $t$. Let $X_{i}(t)=\left(x_{i 1}(t), x_{i 2}(t), . ., x_{i D}(t)\right), x_{i d}(t) \in\{0,1\}$ be binary particle $i$ with $D$ bits at iteration $t . X_{i}(t)$ is the corresponding binary particle of the quantum particle $Q_{i}(t)$ and also can be treated as a potential solution. A binary particle $X_{i}(t)$ can be got from quantum particle $Q_{i}(t)$ by performing a random observation as following:

$x_{i d}(t)=\left\{\begin{array}{l}1 \text { if rand }()>q_{i d}(t) \\ 0 \text { otherwise }\end{array}\right.$

Where rand () is a random number selected from a uniform distribution in $[0,1]$. Let $P_{i}(t)=\left(p_{i 1}\right.$ $\left.(t), p_{i 2}(t), \ldots, p_{i D}(t)\right)$ be the best solution that binary particle $X_{i}(t)$ has obtained until iteration $t$, and $P_{g}(t)=\left(p_{g 1}(t), p_{g 2}(t), \ldots, p_{g D}(t)\right)$ be the best solution obtained from $P_{i}(t)$ in the whole swarm at iteration $t$.

$q_{\text {localbest }}(t)=\alpha \cdot P_{i d}(t)+\beta \cdot\left(1-P_{i d}(t)\right)$

$q_{\text {golbalbest }}(t)=\alpha \cdot P_{\text {id }}(t)+\beta \cdot\left(1-P_{\text {id }}(t)\right)$

$q_{\text {id }}(t+1)=c_{1} \cdot q_{\text {id }}(t)+c_{2} \cdot q_{\text {localbest }}(t)+c_{3} \cdot q_{\text {globalbest }}(t)$

Where $\alpha+\beta=1,0<\alpha, \beta<1$ are control parameters. The smaller of $\alpha$, the bigger of the appear probability of the desired item. $c_{1}+c_{2}+c_{3}=1,0<c_{1}, c_{2}, c_{3}<1$ represent the degree of the belief on oneself, local best solution and global best solution, respectively. In order to keep the diversity in particle swarm and further improve QPSO performance, we incorporated a mutation operator into the QPSO. The mutation operator independently changes the Q-bit of an individual with a mutation probability $p$ as following:

$q_{i d}(t)=1-q_{i d}(t)$, if $\operatorname{rand}()<p$

\section{Genetic Particle Swarm Optimization (GPSO)}

Denote by $N$ the number of particles in the swarm. The GPSO with genetic recombination for the $d$-th bit of particle $i$ is described as follows:

$x_{i d}(t+1)=w\left(0, w_{1}\right) \operatorname{rand}\left(x_{i d}(t)\right)+w\left(w_{1}, w_{2}\right) \operatorname{rand}\left(p_{i d}(t)\right)+w\left(w_{2}, 1\right) \operatorname{rand}\left(p_{g d}(t)\right)$

where $0<w_{1}<w_{2}<1, w()$ and $\operatorname{rand}()$ are a threshold function and a probabilistic bit flipping function, respectively, and they are defined as follows:

$w(a, b)=\left\{\begin{array}{l}1 \text { if } a \leq r_{1} \leq P_{m} \\ 0 \text { otherwise }\end{array}\right.$ 
$\operatorname{rand}(y)=\left\{\begin{array}{l}1-y \text { if } r_{2} \leq p_{m} \\ y \text { otherwise }\end{array}\right.$

Where $r_{1}$ and $r_{2}$ are the random numbers uniformly distributed in [0,1]. Thus, only one of the three terms on right hand side of Eq. (15) will remain dependent on the value $r_{1}$, and $\operatorname{rand}(y)$ mutates the binary bit $y$ with a small mutation probability $\mathrm{pm}$. The updating rule of the genetic PSO is analogue to the genetic algorithm in two aspects. First, the particle derives its single bit from the particle $x_{i d}, p_{i d}$ and $p_{g d}$. This operation corresponds to a 3-way uniform crossover among $X_{i}, P_{i}$ and $P_{g}$, such that the particle can exchange building blocks (segments of ordering or partial selections of elements) with personal and global experiences. Second, each bit attained in this way will be flipped with a small probability $p_{m}$, corresponding to the binary mutation performed in genetic algorithms. As such, genetic reproduction, in particular, crossover and mutation, has been added to the particle swarm optimization. This new genetic version, named GPSO, is very likely more suitable for solving combinatorial optimization problems than the original one.

\section{Improved Quantum Algorithm (IQA)}

It is concluded from "No Free Lunch" theorem [25] that there is no any method can solve all the problems optimally, so that hybrid optimization algorithms have gained wide research in recent years [26, 27]. Based on the description of last section, it can be seen that it is quite different between QPSO and GPSO in terms of representation and evolution operators. In QPSO, the representation of population is Q-bit and evolutionary search is in micro-space (Q-bit based representation space). Differently, in GPSO the representation is binary number and evolutionary search is in macro-space (binary space). We consider the hybridization of QPSO and GPSO to develop hybrid QPSO characterized the principles of both quantum computing and evolutionary computing mechanisms.

Algorithm for solving reactive power dispatch problem.

1. Initialize.

1.1 Set $t=0$, and initialize the $Q P(t)$.

1.2 Make $B P(t)$ by observing the states of $Q P(t)$.

1.3 Evaluate the $B P(t)$, and update the local best solutions and the global best solution.

1.4 Store $B P(t)$ into Parent $(t)$.

2. Repeat until a given maximal number of iterations (MaxIter) is achieved.

2.1 Set $t=t+1$.

2.2 Update $Q P(t)$ using QPSO.

2.3 Make $B P(t)$ by observing the states of $Q P(t)$.

2.4 Evaluate the $B P(t)$.

2.5 Select better one between $B P(t)$ and Parent $(t-1)$ for each individual to update $B P(t)$.

2.6 Update the local best solutions and the global best solution.

2.7 Update $B P(t)$ using GPSO for a given maximal number of iteration (gMaxIter).

2.8 Evaluate the $B P(t)$, and update the local best solutions and the global best solution. 
In the main loop the above procedure, firstly, quantum swarm is evolved by the evolution mechanism of the QPSO (Step 2.2). After one generation evolution of quantum swarm, a random observation is performed on quantum swarm (Step 2.3). Thus, binary swarm is made by the random observation and prepares to be evolved by the evolution mechanism of the GPSO in succession. Note that the individuals to perform GPSO are based on all the individuals resulted by QPSO in current generation and all the individuals resulted by GPSO in last generation (Step 2.5). That is, if a binary individual in the population resulted by QPSO in current generation is worse than the corresponding binary individual in the population resulted by GPSO in last generation, then the worse one is replaced by the better one. This selection process is something like the $(\mu+\lambda)$ selection in evolutionary algorithm [28]. The selection in the hybrid algorithm is helpful to reserve better solutions and speed up the evolution process. After the one or more generation GPSO evolution of binary swarm (Step 2.7), the best solutions that each particle has obtained and the best solution that obtained from the whole swarm are recorded and transferred to quantum swarm to guide a new generation evolution of quantum swarm (Step 2.8). In the hybrid algorithm, the best solutions that each binary particle has obtained and global best solution of whole swarm can also be considered as additional swarm individuals. They not only guide the evolution of quantum swarm, but also guide evolution of binary swarm observed from quantum swarm. Therefore, quantum swarm co-evolves with binary swarm and the information of evolution is exchanged between them by the best solutions and global best solution. With the hybridization of different representation spaces and various particle swarm optimization operators, it can not only enrich the searching behaviour but also enhance and balance the exploration and exploitation abilities to avoid being trapped in local optima. Moreover, to balance the effort of QPSO and GPSO, different parameters can be used, such as population size. On the other hand, the initial inspiration for the PSO was the coordinated movement of swarms of animals in nature, for example schools of fish or flocks of birds. It reflects the cooperative relationship among the individuals within a swarm. However, in natural ecosystems, many species have developed cooperative interactions with other species to improve their survival. Such cooperative co-evolution is called symbiosis [29]. According to the different symbiotic interrelationships, symbiosis can be classified into three main categories: mutualism (both species benefit by the relationship), commensalism (one species benefits while the other species is not affected), and parasitism (one species benefits and the other is harmed) [30]. The coevolution between quantum swarm and binary swarm in the proposed hybrid algorithm is similar to the mutualism model, where both swarms benefit from each other.

\section{Simulation Results}

At first Improved Quantum Algorithm (IQA) has been tested in standard IEEE-57 bus power system. The reactive power compensation buses are 18, 25 and 53. Bus 2, 3, 6, 8, 9 and 12 are PV buses and bus 1 is selected as slack-bus. The system variable limits are given in Table 1.

The preliminary conditions for the IEEE-57 bus power system are given as follows:

$\mathrm{P}_{\text {load }}=12.110$ p.u. $\mathrm{Q}_{\text {load }}=3.031$ p.u.

The total initial generations and power losses are obtained as follows:

$\sum P_{G}=12.4521$ p.u. $\sum Q_{G}=3.3128$ p.u.

$\mathrm{P}_{\text {loss }}=0.25801$ p.u. $\mathrm{Q}_{\text {loss }}=-1.2072$ p.u.

Table 2 shows the various system control variables i.e. generator bus voltages, shunt capacitances and transformer tap settings obtained after optimization which are within the 
acceptable limits. In Table 3, shows the comparison of optimum results obtained from proposed methods with other optimization techniques. These results indicate the robustness of proposed approaches for providing better optimal solution in case of IEEE-57 bus system.

Table 1: Variable Limits

\begin{tabular}{|c|c|c|c|c|c|c|c|}
\hline \multicolumn{8}{|c|}{ Reactive Power Generation Limits } \\
\hline Bus no & 1 & 2 & 3 & 6 & 8 & 9 & 12 \\
\hline Qgmin & -1.4 & -.015 & -.02 & -0.04 & -1 & -0.03 & -0.4 \\
\hline Qgmax & 1 & 0.3 & 0.4 & 0.21 & 1 & 0.04 & 1.50 \\
\hline \multicolumn{8}{|c|}{ Voltage And Tap Setting Limits } \\
\hline vgmin & Vgmax & \multicolumn{2}{|c|}{ vpqmin } & \multicolumn{2}{|c|}{ Vpqmax } & tkmin & tkmax \\
\hline 0.9 & 1.0 & \multicolumn{2}{|c|}{0.91} & \multicolumn{2}{|c|}{1.05} & 0.9 & 1.0 \\
\hline \multicolumn{5}{|c|}{ Shunt Capacitor Limits } & & & \\
\hline Bus no & \multicolumn{3}{|c|}{\begin{tabular}{|l|l|}
18 & 25 \\
\end{tabular}} & \multicolumn{2}{|l|}{53} & & \\
\hline Qcmin & \multicolumn{2}{|l|}{0} & 0 & \multicolumn{2}{|l|}{0} & & \\
\hline Qcmax & 10 & \multicolumn{2}{|c|}{5.2} & \multicolumn{2}{|l|}{6.1} & & \\
\hline
\end{tabular}

Table 2: Control variables obtained after optimization

\begin{tabular}{|l|l|}
\hline Control Variables & IQA \\
\hline V1 & 1.1 \\
\hline V2 & 1.032 \\
\hline V3 & 1.031 \\
\hline V6 & 1.029 \\
\hline V8 & 1.025 \\
\hline V9 & 1.009 \\
\hline V12 & 1.019 \\
\hline Qc18 & 0.0665 \\
\hline Qc25 & 0.200 \\
\hline Qc53 & 0.0471 \\
\hline T4-18 & 1.009 \\
\hline T21-20 & 1.048 \\
\hline T24-25 & 0.868 \\
\hline T24-26 & 0.872 \\
\hline T7-29 & 1.059 \\
\hline T34-32 & 0.879 \\
\hline T11-41 & 1.019 \\
\hline T15-45 & 1.039 \\
\hline T14-46 & 0.910 \\
\hline T10-51 & 1.020 \\
\hline T13-49 & 1.060 \\
\hline T11-43 & 0.910 \\
\hline T40-56 & 0.900 \\
\hline T39-57 & 0.950 \\
\hline T9-55 & 0.950 \\
\hline & \\
\hline
\end{tabular}


Table 3: Comparison results

\begin{tabular}{|l|l|l|l|l|}
\hline S.No. & Optimization Algorithm & Finest Solution & Poorest Solution & Normal Solution \\
\hline 1 & NLP [31] & 0.25902 & 0.30854 & 0.27858 \\
\hline 2 & CGA [31] & 0.25244 & 0.27507 & 0.26293 \\
\hline 3 & AGA [31] & 0.24564 & 0.26671 & 0.25127 \\
\hline 4 & PSO-w [31] & 0.24270 & 0.26152 & 0.24725 \\
\hline 5 & PSO-cf [31] & 0.24280 & 0.26032 & 0.24698 \\
\hline 6 & CLPSO [31] & 0.24515 & 0.24780 & 0.24673 \\
\hline 7 & SPSO-07 [31] & 0.24430 & 0.25457 & 0.24752 \\
\hline 8 & L-DE [31] & 0.27812 & 0.41909 & 0.33177 \\
\hline 9 & L-SACP-DE [31] & 0.27915 & 0.36978 & 0.31032 \\
\hline 10 & L-SaDE [31] & 0.24267 & 0.24391 & 0.24311 \\
\hline 11 & SOA [31] & 0.24265 & 0.24280 & 0.24270 \\
\hline 12 & LM [32] & 0.2484 & 0.2922 & 0.2641 \\
\hline 13 & MBEP1 [32] & 0.2474 & 0.2848 & 0.2643 \\
\hline 14 & MBEP2 [32] & 0.2482 & 0.283 & 0.2592 \\
\hline 15 & BES100 [32] & 0.2438 & 0.263 & 0.2541 \\
\hline 16 & BES200 [32] & 0.3417 & 0.2486 & 0.2443 \\
\hline 17 & Proposed IQA & 0.22086 & 0.23108 & 0.22106 \\
\hline
\end{tabular}

Then Improved Quantum Algorithm (IQA) has been tested in standard IEEE 118-bus test system [33].The system has 54 generator buses, 64 load buses, 186 branches and 9 of them are with the tap setting transformers. The limits of voltage on generator buses are $0.95-1.1$ per-unit., and on load buses are $0.95-1.05$ per-unit. The limit of transformer rate is $0.9-1.1$, with the changes step of 0.025 . The limitations of reactive power source are listed in Table 4, with the change in step of 0.01 .

Table 4: Limitation of reactive power sources

\begin{tabular}{|l|l|l|l|l|l|l|l|}
\hline BUS & 5 & 34 & 37 & 44 & 45 & 46 & 48 \\
\hline QCMAX & 0 & 14 & 0 & 10 & 10 & 10 & 15 \\
\hline QCMIN & -40 & 0 & -25 & 0 & 0 & 0 & 0 \\
\hline BUS & 74 & 79 & 82 & 83 & 105 & 107 & 110 \\
\hline QCMAX & 12 & 20 & 20 & 10 & 20 & 6 & 6 \\
\hline QCMIN & 0 & 0 & 0 & 0 & 0 & 0 & 0 \\
\hline
\end{tabular}

The statistical comparison results of 50 trial runs have been list in Table 5 and the results clearly show the better performance of proposed Improved Quantum Algorithm (IQA) in reducing the real power loss.

Table 5: Comparison results

\begin{tabular}{|l|l|l|l|l|}
\hline Active power loss (MW) & BBO [34] & $\begin{array}{l}\text { ILSBBO/ } \\
\text { strategy1 [34] }\end{array}$ & $\begin{array}{l}\text { ILSBBO/ } \\
\text { strategy1 [34] }\end{array}$ & Proposed IQA \\
\hline Min & 128.77 & 126.98 & 124.78 & 117.90 \\
\hline Max & 132.64 & 137.34 & 132.39 & 121.97 \\
\hline Average & 130.21 & 130.37 & 129.22 & 120.01 \\
\hline
\end{tabular}




\section{Conclusion}

In this paper Combination of the Q-bit evolutionary search - quantum particle swarm optimization (QPSO) algorithm and binary bit evolutionary search - genetic particle swarm optimization (GPSO) termed as Improved Quantum Algorithm (IQA) has been used to solve reactive power dispatch problem. The effectiveness of the proposed method has been demonstrated by testing it in IEEE 57,118 bus system and simuation results reveals about the reduction of real power loss when compared with other standard algorithms and also volatge profiles are within the limits .

\section{References}

[1] O.Alsac,and B. Scott, "Optimal load flow with steady state security",IEEE Transaction. PAS 1973, pp. 745-751.

[2] Lee K Y ,Paru Y M , Oritz J L -A united approach to optimal real and reactive power dispatch , IEEE Transactions on power Apparatus and systems 1985: PAS-104 : 1147-1153

[3] A.Monticelli , M .V.F Pereira ,and S. Granville, "Security constrained optimal power flow with post contingency corrective rescheduling", IEEE Transactions on Power Systems :PWRS-2, No. 1, pp.175-182.,1987.

[4] Deeb N ,Shahidehpur S.M ,Linear reactive power optimization in a large power network using the decomposition approach. IEEE Transactions on power system 1990: 5(2) : 428-435

[5] E. Hobson ,'Network consrained reactive power control using linear programming, ' IEEE Transactions on power systems PAS -99 (4) ,pp 868-877, 1980

[6] K.Y Lee ,Y.M Park, and J.L Oritz, "Fuel -cost optimization for both real and reactive power dispatches", IEE Proc; 131C,(3), pp.85-93.

[7] M.K. Mangoli, and K.Y. Lee, "Optimal real and reactive power control using linear programming”, Electr.Power Syst.Res, Vol.26, pp.1-10,1993.

[8] S.R.Paranjothi , and K.Anburaja, "Optimal power flow using refined genetic algorithm", Electr.Power Compon.Syst, Vol. 30, 1055-1063,2002.

[9] D. Devaraj, and B. Yeganarayana, "Genetic algorithm based optimal power flow for security enhancement", IEE proc-Generation.Transmission and. Distribution; 152, 6 November 2005.

[10] C.A. Canizares , A.C.Z.de Souza and V.H. Quintana , "Comparison of performance indices for detection of proximity to voltage collapse ,' vol. 11. no.3 , pp.1441-1450, Aug 1996.

[11] $\mathrm{Wu} \mathrm{Q} \mathrm{H}$, Ma J T. Power system optimal reactive power dispatch using evolutionary programming. IEEE Transactions on power systems 1995; 10(3): 1243-1248 .

[12] S.Durairaj, D.Devaraj, P.S.Kannan ,' Genetic algorithm applications to optimal reactive power dispatch with voltage stability enhancement', IE(I) Journal-EL Vol 87,September 2006.

[13] D.Devaraj ,' Improved genetic algorithm for multi - objective reactive power dispatch problem' European Transactions on electrical power 2007 ; 17: 569-581.

[14] P. Aruna Jeyanthy and Dr. D. Devaraj "Optimal Reactive Power Dispatch for Voltage Stability Enhancement Using Real Coded Genetic Algorithm" International Journal of Computer and Electrical Engineering, Vol. 2, No. 4, August, 2010 1793-8163.

[15] Kennedy, J. \& Eberhart, R.C. (1995) Particle swarm optimization. Proceedings of IEEE International Conference on Neural Networks: 1942-1948. NJ: Piscataway.

[16] Van den Bergh, F. \& Engelbrecht, A.P. (2000) Cooperative learning in neural network using particle swarm optimizers. South African Computer Journal 26: 84-90,.

[17] El-Galland, AI., El-Hawary, ME. \& Sallam, AA. (2001) Swarming of intelligent particles for solving the nonlinear constrained optimization problem. Engineering Intelligent Systems for Electrical Engineering and Communications 9: 155-163. 
[18] Parsopoulos, K.E. \& Vrahatis, M.N. (2002) Recent approaches to global optimization problems through Particle Swarm Optimization. Natural Computing 1(2-3): 235-306.

[19] Kennedy, J. \& Eberhart, R.C. (1997) A discrete binary version of the particle swarm algorithm. Proceedings of the World Multiconference on Systemics, Cybernetics and Informatics: 41044109. NJ: Piscatawary.

[20] Franken, N. \& Engelbrecht, A.P. (2005) Investigating binary PSO parameter influence on the knights cover problem. IEEE Congress on Evolutionary Computation 1: 282-289.

[21] Huang, Y.-X., Zhou, C.-G., Zou, S.-X. \& Wang, Y. (2005) A hybrid algorithm on class cover problems. Journal of Software (in Chinese) 16(4): 513-522.

[22] Yang, S.Y., Wang, M. \& Jiao, L.C. (2004) A quantum particle swarm optimization. Proceeding of the 2004 IEEE Congress on Evolutionary Computation 1: 320-324,.

[23] Yin, P.Y. (2006) Genetic particle swarm optimization for polygonal approximation of digital curves. Pattern Recognition and Image Analysis 16(2): 223-233,.

[24] Han, K.H. \& Kim, J.H. (2002) Quantum-inspired evolutionary algorithm for a class of combinatorial optimization. IEEE Transactions on Evolutionary Computation 6(6): 580-593.

[25] Wolpert, D.H. \& Macready, W.G. (1997) No free lunch theorems for optimization. IEEE Transactions on Evolutionary Computation 1(1): 67-82.

[26] Wang, L. \& Zheng, D.Z. (2001) An effective hybrid optimization strategy for job-shop scheduling problems. Computers and Operations Research 28: 585-596.

[27] Wang, L., Tang, F. \& Wu, H. (2005) Hybrid genetic algorithm based on quantum computing for numerical optimization and parameter estimation. Applied Mathematics and Computation 171: 1141-1156.

[28] Back, T. (1994) Selective pressure in evolutionary algorithm: a characterization of selection mechanisms. Proceeding of the first IEEE Conference on Evolutionary Computation, IEEE Press: 57-62. Piscataway, HJ.

[29] Ahmadjian, V. \& Paracer, S. (2000) Symbiosis: An Introduction to Biological Associations. Oxford University Press, New York.

[30] Douglas, A.E. (1994) Symbiotic Interactions. Oxford University Press, Oxford.

[31] Chaohua Dai, Weirong Chen, Yunfang Zhu, and Xuexia Zhang, "Seeker optimization algorithm for optimal reactive power dispatch," IEEE Trans. Power Systems, Vol. 24, No. 3, August 2009, pp. 1218-1231.

[32] J. R. Gomes and 0. R. Saavedra, "Optimal reactive power dispatch using evolutionary computation: Extended algorithms," IEE Proc.-Gener. Transm. Distrib.. Vol. 146, No. 6. Nov. 1999.

[33] IEEE, "The IEEE 30-bus test system and the IEEE 118-test system", (1993), http://www.ee.washington.edu/trsearch/pstca/.

[34] Jiangtao Cao, Fuli Wang and Ping Li, "An Improved Biogeography-based Optimization Algorithm for Optimal Reactive Power Flow" International Journal of Control and Automation Vol.7, No.3 (2014), pp.161-176.

*Corresponding author.

E-mail address: gklenin@gmail.com 